RUNNING HEAD: THEORETICAL EXPLANATIONS

\title{
Strengths and limitations of theoretical explanations in psychology
}

\author{
Jan De Houwer \\ Ghent University, Ghent, Belgium \\ Klaus Fiedler \\ University of Heidelberg, Heidelberg, Germany \\ and \\ Agnes Moors \\ Ghent University, Ghent, Belgium
}

In Press. Perspectives on Psychological Science.

mailing address:

Jan De Houwer

Ghent University

Henri Dunantlaan 2

B-9000 Ghent

Belgium

email: Jan.DeHouwer@UGent.be

phone: 003292646445

fax: 003292646489 


\section{Strengths and limitations of theoretical explanations in psychology}

The current state of the arts in psychological science can be described as the end of an era of hunting and gathering empirical findings. Psychologists have been extremely successful and industrious during this period of empirical research. We believe that it is now time to focus more extensively on theoretical explanations. The pluralism of methods and the increasingly interdisciplinary nature of psychological research call for more refined and methodologically sound theoretical explanations that go beyond circular accounts and labeling-type interpretations expressed in ordinary language.

In order to take this important next step, we need to specify the criteria that sound explanations should meet, to reveal the various reasons for why current theories often do not meet those criteria, and to exchange ideas about how current theoretical practice can be improved. This special section of the Journal "Perspectives on Psychological Science" contains five papers in which researchers from various sub-disciplines in psychology reflect on these issues.

Theorizing is guided by data and can thus be biased by selectivity in the collection of data. Fiedler (this issue) argues that selectivity in data collection is omnipresent in psychological science. More often than not, hypotheses about what the data should look like are used to guide the selection of data that are used to test those hypotheses. Whereas the recent high-profile debate on "voodoo-correlations" revealed one instance of this practice in neuroscience, Fiedler reveals many other common instances of hypothesis-driven selectivity in data collection. Although selectivity in data collection does not necessarily invalidate the data as such, it is vital for scientific progress that the nature of the selectivity is made explicit and that data are collected across a wider range of situations.

Selectivity in data collection is often linked to the concept of circularity. Hahn (this 
issue) provides a highly insightful analysis of what it actually means to say that arguments, explanations, data analyses, and research methods are circular. Based on the analysis, she concludes that the problems of circularity should not be overstated. On the one hand, some types of circular arguments and explanations can improve scientific understanding. On the other hand, claims about circular data analysis and methods often do not refer to actual instances of circularity but are related more to a failure to make explicit selectivity in the manner in which data have been collected.

Selectivity in the collection of data is exacerbated by the strong focus in psychological science on paradigm-specific methods and effects. As Meiser (this issue) correctly points out, research and theorizing often deals primarily with effects in one particular experimental paradigm. Importantly, selectivity in data selection leads to selectivity in theorizing. It narrows the universality of theories, renders it more difficult to discriminate between different theories, and hinders the search for mechanisms that operate in a variety of situations. Therefore, more attempts should be made to develop theories that try to explain behavior in a variety of paradigms and situations.

The paper of Garcia-Marques and Ferreira (this issue) also deals with selectivity at the theoretical level. Many theories are built on a theoretical dichotomy, that is, on one of two complementing or opposing processes (e.g., implicit vs. explicit memory) or representations (e.g. modal vs. amodal representations). Because of the focus on a dichotomy, the models tend to be imprecise and thus flexible in their assumptions about the other types of processes or representations that are needed to provide a full account of behavior. As a result, various models become indistinguishable, leading to the abandonment of the theoretical dichotomy on which the models were built. For instance, given certain assumptions about how representations influence behavior, models that feature modal 
representations can mimic the output of models with amodal representations and vice versa. Garcia-Marques and Ferreira call for more high-level theories that go beyond simple dichotomies and can be applied to a broader range of phenomena. However, they acknowledge that also high-level cognitive theories can be difficult to evaluate empirically. Nevertheless, theoretical progress can be achieved because experimental data put limits on the types of cognitive theories that can be put forward. This type of progress can be optimized by clearly separating data from the theories used to explain these data.

De Houwer (this issue) also calls for a clear separation of the explanandum (that which needs to be explained) and the explanans (that which is used to explain). This important scientific principle is violated when an empirical effect (e.g., negative priming) is used as a proxy for a cognitive process (e.g., inhibition). He discusses different ways in which these violations can hamper scientific progress. In order to guard cognitive research against these violations, De Houwer proposes a functional-cognitive approach in which the cognitive approach is grafted on a functional approach. The functional approach aims to uncover the environmental causes of behavior and does so without referring to mental constructs. The aim of the cognitive approach is to uncover the mental processes that mediate the impact of the environment on behavior. The approaches are mutually supportive in that the functional approach provides the input for the cognitive approach whereas the cognitive approach can help organize and guide functional research.

Although all contributors discuss problems in current scientific practice, they are also constructive in that they put forward possible solutions for these problems and discuss the boundaries of the problems they have identified. With regard to the latter point, an important message that emanates from the papers is the idea that the criteria used to judge the quality of research and theorizing depends on the goals of the researcher. For instance, selectivity in 
data analysis, in the scope of theories, and in the constructs on which theories are built might hamper theory testing but promote the discovery of new empirical effects. It is probably because of the advantage for the goal of exploration that potentially problematic scientific practices such as selectivity in data analysis have been so popular in the recent era of hunting and gathering empirical findings. Rather than abandoning these practices completely and replacing them by the alternatives put forward by the contributors of this special section, one should become aware of their limitations and be open for alternatives that can overcome these limitations. We hope that this special section will help researchers to reflect on their scientific practice in this manner. 


\section{References}

De Houwer, J. (this issue). Why the cognitive approach in psychology would profit from a functional approach and vice versa. Perspectives in Psychological Science.

Fiedler, K. (this issue). Voodoo correlations - A severe methodological problem, not only in social neurosciences. Perspectives in Psychological Science.

Garcia-Marques, L., \& Ferreira, M. B. (this issue). Friends and foes of theory construction in psychological science: Vague dichotomies, unified theories of cognition and the new experimentalism. Perspectives in Psychological Science.

Hahn, U. (this issue). The problem of circularity in evidence, argument and explanation. Perspectives in Psychological Science.

Krueger, J. I. (this issue). Beyond comparisons in research and reasoning. Perspectives in Psychological Science.

Meiser, T. (this issue). Much pain, little gain? Paradigm-specific models and methods in experimental psychology. Perspectives in Psychological Science. 


\section{Author Note}

Jan De Houwer and Agnes Moors, Ghent University, Ghent, Belgium; Klaus Fiedler, University of Heidelberg, Heidelberg, Germany The preparation of this paper was made possible by Grants BOF/GOA2006/001 and BOF09/01M00209 of Ghent University and by Travel Grant K142406N01 of the Research Foundation-Flanders (FWO-Vlaanderen, Belgium). Correspondence should be addressed to Jan De Houwer, Ghent University, Henri Dunantlaan 2, B-9000 Ghent, Belgium. Electronic mail can be sent to Jan.DeHouwer@UGent.be . 